\title{
New country and host records for Lithuanian Tachinidae (Diptera)
}

\section{Erikas Lutovinovas}

Lutovinovas, E. 2012: New country and host records for Lithuanian Tachinidae (Diptera). - Entomol. Fennica 23: 231-238.

This paper updates the knowledge on the family Tachinidae (Diptera) in the Lithuanian fauna. As the result, 68 species are first records from the country and eight species are deleted from the previous list. Among species listed in this paper, 54 represent first records for the Baltic States and 16 are new for all of Eastern Europe. Parasitoid-host associations for 13 species of Tachinidae with 15 host taxa that comprise 17 couples are recorded for Lithuania for the first time. Among these, Meigenia uncinatata is a new parasitoid of the leaf beetle Gonioctena quinquepunctata (Chrysomelidae).

E. Lutovinovas, Department of Botany and Zoology, Masaryk University, Kotlářská 2, CZ-61137Brno, Czech Republic; E-mail:wohlfahrtia@gmail.com

Received 5 September 2011, accepted 28 November 2011

\section{Introduction}

Tachinid flies are a worldwide distributed family of two-winged insects, related to botflies, blowflies and several other families having a negative reputation from the medical and veterinary point of view. Their representatives are rather diverse in body size and coloration, from black and tiny, rarely seen, to bright and large, easily spotted in the field. It is one of the largest families in the order, being more prevalent in natural habitats, whereas poorly observed in the areas affected by human activities. The latter fact may have important implications for their utilisation as bioindicators of well-preserved habitats (Tooker et al. 2006). All species are parasitoids of terrestrial arthropods, including many orders of insects, centipedes, arachnids, and therefore regarded beneficial when developing in pests of agriculture and forestry (Tschorsnig et al. 2005).

The family has been insufficiently studied in Lithuania. The aim of the present paper is to provide a deeper knowledge on the tachinid fauna and to make our knowledge of the parasitoid-host associations of this group within the study area more complete.

\section{Material and methods}

Most of the material was collected in 2008, but older records have also been included. The material is chiefly deposited in the private collection of the author. Specimens were either directly sampled in the field or collected with their hosts and then reared in the laboratory. Hosts were either collected from their food-plants as immature stages (moths, sawflies), or from Symmorphus allobrogus (Saussure) predatory wasp nests (beetles); for methods of the collecting of wasp prey from the wasps' nests see Budrienè (2003).

Morphological identification of flies was possible using a combination of sources (Tschorsnig \& Herting 1994, Andersen 1996, Ziegler \& Shima 1996, Ziegler 1999, Bystrowski 2001, Bergström 2007). Host species were identified by the 
collectors of the material. Identification of beetle larvae, as well as complicated females of the Meigenia mutabilis (Fallén) group were additionally checked by a molecular method: portions of mitochondrial rDNA obtained from the exuviae were compared with sequences from the Gene Bank, or with sequences obtained from reliably identified specimens. Nuclear acids extraction and amplification technique followed Lutovinovas et al. (submitted).

The results are presented in several parts. The following list of new records provides faunistic records, host records and corrections of the previous list (Pakalniškis et al. 2006). All faunistic records are new for Lithuania, partly new for the territory of the Baltic States or for the whole Eastern Europe. Full names for the localities are shown; their coordinates and collectors are provided separately (Table 1).

\section{Results}

\subsection{Faunistic records}

Records are marked with an asterisk $\left(^{*}\right)$ if new for the territory of the Baltic States, with two asterisks $(* *)$ if new for the whole Eastern Europe (former USSR). Sampling methods and their abbreviations are as follows: Malaise trap (M), yellow sticky card $(\mathrm{Y})$, hand net $(\mathrm{N})$, pit-fall trap $(\mathrm{P})$, attracted by light $(\mathrm{L})$, or collected with a host $(\mathrm{H})$.

\subsubsection{Subfamily Dexiinae}

*Athrycia impressa (van der Wulp, 1869). Eičiai, 26.V.-30.VI.2008, 7ठえ, M.

Billaea kolomyetzi Mesnil, 1970. Leipgiriai, 30.VI.-09.VII.1999, 1ठ̄, M.

**Billaea steini (Brauer \& Bergenstamm, 1890).

Leipgiriai, 01.-05.VIII.2008, 19, M.

Campylocheta inepta (Meigen, 1824). Dūkštos, 24.VI.-01.VII.2007, 1今, M; Eičiai, 15.21.VII.2008, 19, M; Subartonys, 07.-29.VII. 2008, 3 우, M.

*Campylocheta mariae Bystrowski, 2001.

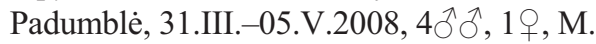

**Campylocheta similis Ziegler \& Shima, 1996.

Paneriai, 16.IV.2004, 1ㅇ, N.

*Cyrtophleba vernalis (Kramer, 1917). Zervynos, 02.-13.V.2010, 1今̄, Y.
*Eriothrix prolixa (Meigen, 1824). Semeliškès, 17.VII.2007, 1ठ, N.

*Hyleorus elatus (Meigen, 1838). Eičiai, 15.21.VII.2008, 10̄, M.

Klugia marginata (Meigen, 1824). Obelija, 28.VI.1980, 1, N.

* Ramonda spathulata (Fallén, 1820). Pamedinčiai, 12.V.-16.VI.2008, 2ふ̂す, M.

*Rondania fasciata (Macquart, 1834). Zervynos, 13.V.-01.VI.2010, 1ठ̄, Y.

*Trixa caerulescens Meigen, 1824. Pamedinčiai, 19.-26.V.2008, 1 , , M.

Wagneria alpina Villeneuve, 1910. Godeliai, 14.21.VII.2008, 1ठ̂, M; Pamedinčiai, 07.14.VII.2008, 1ठ, M.

Wagneria costata (Fallén, 1815). Leipgiriai, 08.15.VII.2008, 1q, M; Padumblè, 30.VI.07.VII.2008, 1 , , M.

\subsubsection{Subfamily Phasiinae}

*Catharosia pygmaea (Fallén, 1815). Godeliai,

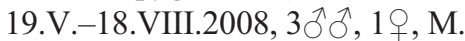

*Cinochira atra Zetterstedt, 1845. Godeliai, 07.14.VII.2008, 2§ิð, 1, M; Pamedinčiai, 23.30.VI.2008, 1今, M; Subartonys, 16.-23.VI. 2008, 1 ㅇ, M.

*Cylindromyia brevicornis (Loew, 1844). Puvočiai, 30.VI.1995, 1, N.

* Gymnosoma dolycoridis Dupuis, 1961. Balbieriškis, 17.VIII.2006, 1ð̄, N; Jūrè, 09.VII. 1998, 1§̃, N.

*Redtenbacheria insignis Egger, 1861. Verkiai, 04.VII.2005, 1q, L.

\subsubsection{Subfamily Tachininae}

*Aphantorhaphopsis samarensis (Villeneuve, 1921). Leipgiriai, 27.V.-30.VI.2008, 5ठえ Subartonys, 02.VI.-28.VII.2008, 3§ิð, 8우우, M.

*Aphantorhaphopsis verralli (Wainwright, 1928). Padumble, 21.-28.VII.2008, 2ðð, M.

*Ceranthia lichtwardtiana (Villeneuve, 1931).

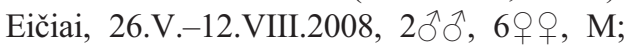
Godeliai, 19.V.-18.VIII.2008, 4우, M; Leipgiriai, 27.V.-02.VI.2008, 2 우, M; Markučiai, 02.-09.VIII.2004, 1ठ, M; Pamedinčiai, 19.V.04.VIII.2008, 4ภํ, 8우, M; Subartonys,

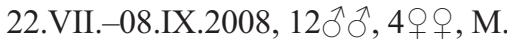


Table 1. Sampling locations with collectors of Tachinidae in Lithuania.

\begin{tabular}{|c|c|c|c|c|}
\hline No & Locality & Administrative unit & Coordinate & Collector \\
\hline 1 & Airènai & Vilnius distr. & $54^{\circ} 5100^{\prime \prime} \mathrm{N} 24^{\circ} 5700^{\prime \prime} \mathrm{E}$ & Lutovinovas, E. \\
\hline 2 & Aukštadvaris & Trakai distr. & $54^{\circ} 3450^{\prime \prime} \mathrm{N} 24^{\circ} 3140^{\prime \prime} \mathrm{E}$ & Lutovinovas, E. \\
\hline 3 & Balbieriškis & Prienai distr. & $54^{\circ} 3050^{\prime \prime} \mathrm{N} 23^{\circ} 5330^{\prime \prime} \mathrm{E}$ & Lutovinovas, E. \\
\hline 4 & Bilšiai & Molètai distr. & $55^{\circ} 0759^{\prime \prime} \mathrm{N} 25^{\circ} 1616$ "E & Budrys, E. \\
\hline 5 & Čižiūnai & Trakai distr. & $54^{\circ} 3540^{\prime \prime} \mathrm{N} 24^{\circ} 3400^{\prime \prime} \mathrm{E}$ & Lutovinovas, E. \\
\hline 6 & Druskininkai & Druskininkai munic. & $54^{\circ} 0110^{\prime \prime} \mathrm{N} 23^{\circ} 5820 " \mathrm{E}$ & Gedminas, A. \\
\hline 7 & Dūkštos & Vilnius distr. & $54^{\circ} 4920^{\prime \prime} \mathrm{N} 24^{\circ} 5810^{\prime \prime} \mathrm{E}$ & Lutovinovas, E. \\
\hline 8 & Eičiai & Tauragè distr. & $55^{\circ} 1050^{\prime \prime} \mathrm{N} 22^{\circ} 2900 ” \mathrm{E}$ & Uselis, V. \\
\hline 9 & Eitminiškès & Vilnius distr. & $54^{\circ} 5520^{\prime \prime} \mathrm{N} 25^{\circ} 2320^{\prime \prime} \mathrm{E}$ & Ivinskis, $\mathrm{P}$. \\
\hline 10 & Godeliai & Plungè distr. & $55^{\circ} 5859^{\prime \prime} \mathrm{N} 21^{\circ} 4713^{\prime \prime} \mathrm{E}$ & Kvašinskas, S. \\
\hline 11 & Griškabūdis & Šakiai distr. & $54^{\circ} 5110^{\prime \prime} \mathrm{N} 23^{\circ} 1000^{\prime \prime} \mathrm{E}$ & Karalius, V. \\
\hline 12 & Gruodžiai & Molètai distr. & $55^{\circ} 1610^{\prime \prime} \mathrm{N} 25^{\circ} 1600 " \mathrm{E}$ & Lutovinovas, E. \\
\hline 13 & Jūrè & Kazlų Rūda munic. & $54^{\circ} 4530^{\prime \prime} \mathrm{N} 23^{\circ} 3540^{\prime \prime} \mathrm{E}$ & Meržijevskis, A. \\
\hline 14 & Karkazai & Šakiai distr. & $55^{\circ} 0437^{\prime \prime} \mathrm{N} 23^{\circ} 1200 " \mathrm{E}$ & Rimšaitè, J. \\
\hline 15 & Kleboniškis & Kaunas munic. & $54^{\circ} 5710^{\prime \prime} \mathrm{N} 23^{\circ} 5650^{\prime \prime} \mathrm{E}$ & Dumčius, $\mathrm{O}$. \\
\hline 16 & Lavysas & Varèna distr. & $54^{\circ} 0950^{\prime \prime} \mathrm{N} 24^{\circ} 2559^{\prime \prime} \mathrm{E}$ & Gedminas, A. \\
\hline 17 & Leipgiriai & Jurbarkas distr. & $55^{\circ} 0619^{\prime \prime} \mathrm{N} 22^{\circ} 2716^{\prime \prime} \mathrm{E}$ & Uselis, V. \\
\hline 18 & Lekéčiai & Šakiai distr. & $54^{\circ} 5900^{\prime \prime} \mathrm{N} 23^{\circ} 3000^{\prime \prime E}$ & Ferenca, R. \\
\hline 19 & Liškiava & Varèna distr. & $54^{\circ} 0450^{\prime \prime} \mathrm{N} 24^{\circ} 0320^{\prime \prime} \mathrm{E}$ & Nedveckytè, I. \\
\hline 20 & Marimantas & Zarasai distr. & $55^{\circ} 4919^{\prime \prime} \mathrm{N} 25^{\circ} 5425^{\prime \prime} \mathrm{E}$ & Ivinskis, $\mathrm{P}$. \\
\hline 21 & Markučiai & Vilnius munic. & $54^{\circ} 40^{\prime} 21^{\prime \prime N} 25^{\circ} 18^{\prime} 58^{\prime \prime E}$ & Lutovinovas, E. \\
\hline 22 & Meteliai & Lazdijai distr. & $54^{\circ} 1810^{\prime \prime} \mathrm{N} 23^{\circ} 4440 " \mathrm{E}$ & Kondratavičiūtè \\
\hline 23 & Mištautai & Zarasai distr. & $55^{\circ} 3947^{\prime \prime} \mathrm{N} 26^{\circ} 3549^{\prime \prime} \mathrm{E}$ & Ivinskis, $P$. \\
\hline 24 & Nida & Neringa munic. & 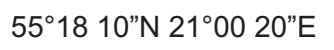 & Gedminas, A. \\
\hline 25 & Noreikiškès & Kaunas distr. & $54^{\circ} 5310^{\prime \prime} \mathrm{N} 23^{\circ} 5050^{\prime \prime} \mathrm{E}$ & Dumčius, $\mathrm{O}$. \\
\hline 26 & Noruliai & Varèna distr. & $54^{\circ} 0640^{\prime \prime} \mathrm{N} 24^{\circ} 1220^{\prime \prime} \mathrm{E}$ & Ivinskis, $\mathrm{P}$. \\
\hline 27 & Obelija & Alytus distr. & $54^{\circ} 1829^{\prime \prime} \mathrm{N} 23^{\circ} 5140^{\prime \prime} \mathrm{E}$ & Švogžlys, P. \\
\hline 28 & Padumblè & Švenčionys distr. & $55^{\circ} 1440^{\prime \prime} \mathrm{N} 25^{\circ} 4710^{\prime \prime} \mathrm{E}$ & Aliukonis, A. \\
\hline 29 & Palkabalis & Varèna distr. & $54^{\circ} 0831^{\prime \prime} \mathrm{N} 24^{\circ} 3640^{\prime \prime} \mathrm{E}$ & Gedminas, A. \\
\hline 30 & Pamedinčiai & Plungè distr. & $56^{\circ} 0126^{\prime \prime} \mathrm{N} 21^{\circ} 46599^{\prime \prime}$ & Kvašinskas, S. \\
\hline 31 & Paneriai & Vilnius munic. & $54^{\circ} 38^{\prime} 27^{\prime \prime N} 25^{\circ} 12^{\prime} 39^{\prime \prime E}$ & Lutovinovas, E. \\
\hline 32 & Purviniškiai & Švenčionys distr. & $55^{\circ} 0159^{\prime \prime} \mathrm{N} 25^{\circ} 3517 " \mathrm{E}$ & Ivinskis, P. \\
\hline 33 & Puvočiai & Varèna distr. & $54^{\circ} 0646^{\prime \prime} \mathrm{N} 24^{\circ} 1818 ” \mathrm{E}$ & llginytè, R. \\
\hline 34 & Sakalinè & Tauragè distr. & $55^{\circ} 1110^{\prime \prime} \mathrm{N} 22^{\circ} 2241^{\prime \prime} \mathrm{E}$ & Uselis, V. \\
\hline 35 & Semeliškès & Elektrènai munic. & $54^{\circ} 4010^{\prime \prime} \mathrm{N} 24^{\circ} 3920^{\prime \prime} \mathrm{E}$ & Lutovinovas, E. \\
\hline 36 & Subartonys & Varèna distr. & $54^{\circ} 1215^{\prime \prime} \mathrm{N} 24^{\circ} 1045^{\prime \prime} \mathrm{E}$ & Lapelè, V. \\
\hline 37 & Šeškinè & Vilnius munic. & $54^{\circ} 4236^{\prime \prime} \mathrm{N} 25^{\circ} 1438$ "E & Lutovinovas, E. \\
\hline 38 & Vartai & Lazdijai distr. & $54^{\circ} 2400^{\prime \prime} \mathrm{N} 23^{\circ} 2940 " \mathrm{E}$ & Lutovinovas, E. \\
\hline 39 & Vebliai & Švenčionys distr. & $54^{\circ} 5349^{\prime \prime} \mathrm{N} 25^{\circ} 4934 " \mathrm{E}$ & Dapkus, D. \\
\hline 40 & Veisiejai & Lazdijai distr. & $54^{\circ} 0600^{\prime \prime} \mathrm{N} 23^{\circ} 4140^{\prime \prime} \mathrm{E}$ & Dumčius, $\mathrm{O}$. \\
\hline 41 & Verkiai & Vilnius munic. & $54^{\circ} 46^{\prime} 60^{\prime \prime} \mathrm{N} 25^{\circ} 21^{\prime} 0 " \mathrm{E}$ & Ivinskis, $\mathrm{P}$. \\
\hline 42 & Visoriai & Vilnius munic. & $54^{\circ} 45^{\prime} 19^{\prime \prime} \mathrm{N} 25^{\circ} 15^{\prime} 55^{\prime \prime E}$ & Lutovinovas, E. \\
\hline 43 & Vyšniūnai & Prienai distr. & $54^{\circ} 3220^{\prime \prime} \mathrm{N} 24^{\circ} 1940^{\prime \prime} \mathrm{E}$ & Lutovinovas, E. \\
\hline 44 & Zervynos & Varèna distr. & $54^{\circ} 0640^{\prime \prime} \mathrm{N} 24^{\circ} 2949^{\prime \prime} \mathrm{E}$ & Gedminas, A. \\
\hline 45 & Žaizdriai & Trakai distr. & $54^{\circ} 3740^{\prime \prime} \mathrm{N} 24^{\circ} 5340^{\prime \prime} \mathrm{E}$ & Lutovinovas, E. \\
\hline 46 & Žaliakalnis & Kaunas munic. & $54^{\circ} 5430^{\prime \prime} \mathrm{N} 23^{\circ} 5530^{\prime \prime} \mathrm{E}$ & Paulavičiūtè, B. \\
\hline 47 & Želsva & Marijampolè munic. & $54^{\circ} 2550^{\prime \prime} \mathrm{N} 23^{\circ} 2410^{\prime \prime} \mathrm{E}$ & Lutovinovas, E. \\
\hline 48 & Želtiškiai & Anykščiai distr. & $55^{\circ} 2400^{\prime \prime} \mathrm{N} 25^{\circ} 1511$ "E & Budrys, E. \\
\hline
\end{tabular}


*Ceranthia pallida Herting, 1959. Subartonys, 21.-28.VII.2008, $2 \hat{\jmath}$ oे, M.

*Ceranthia tenuipalpis (Villeneuve, 1921). Dūkštos, 20.-27.V.2007, 1Ô, M; Eičiai, 12.19.V.2008, 1§, M; Subartonys, 12.V.23.VI.2008, $1{ }^{\Uparrow}, 1$, , M.

Ceromya bicolor (Meigen, 1822). Common form: Eičiai, 08.-14.VII.2008, 1ڤ̂, M; Leipgiriai, 27.V.-02.VI.2008, 1ठ, M. Melanistic form: Eičiai, 01.-05.VIII.2008, 1ㅇ, M; Padumblè, 21.VII.-10.VIII.2008, $2 \widehat{\jmath}$, M.

Note. Melanistic form was previously known only from a type material of $C$. fasciata (Stein) that was synonymised with C. bicolor (Meigen) by Herting (1977). Andersen (1996) has recorded the end of activity of this species before the end of July; melanistic form flies after that in Lithuania (see above).

**Ceromya dorsigera Herting, 1967. Eičiai, 01.05.VIII.2008, 1ㅇ, M; Padumblè, 07.VII.10.VIII.2008, 4えึิ, M; Subartonys, 07.14.VII.2008, 2 $\precsim$, M.

*Ceromya flaviceps (Ratzeburg, 1844). Lavysas, 13.V.-01.VI.2010, $2 \hat{\jmath}$ \%, Y; Palkabalis, 13.V.01.VI.2010, 2§ิठี, Y; Zervynos, 13.V.-

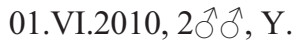

*Chrysosomopsis aurata (Fallén, 1810). Leipgiriai, 15.-22.VII.2008, 1ठ, M; Subartonys, 22.-29.VII.2008, 1 q, M.

**Entomophaga sufferta (Villeneuve, 1942). Airènai, 13.-27.V.2007, 3ठ̂̉ ô, M; Dūkštos, 20.V.-03.VI.2007, 5ठ̂す, M; Pamedinčiai, 05.19.V.2008, 4ふึग, M; Subartonys, 21.28.IV.2008, 10े, M.

Eurithia vivida (Zetterstedt, 1838). Sakalinè, 09.VI.2000, 1 \%, N.

**Goniocera schistacea Brauer \& Bergenstamm, 1891. Godeliai, 28.IV.-12.V.2008, 2 우, M; Želtiškiai, 25.IV.2010, 1今̄, N.

**Loewia erecta Bergström, 2007. Dūkštos, 01.08.VII.2007, 1 ㅇ, M.

* Macquartia pubiceps (Zetterstedt, 1845). Eičiai,

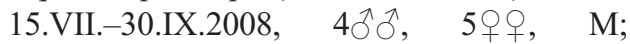

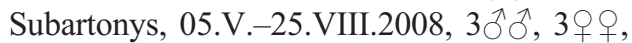
M.

**Peribaea longirostris Andersen, 1996. Airènai, 09.-16.IX.2007, 1, M; Eičiai, 09.VI.08.IX.2008, 159ðึ, 81우，M; Leipgiriai, 27.V.-19.VIII.2008， 24えึิ，14우， M; Meteliai, 30.VII.-06.VIII.2008, 1ठิ, M;

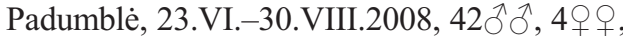
M; Subartonys, 02.VI.-08.IX.2008, 98 $\widehat{\partial}$, 18 우,, M; Vartai, 18.VIII.2006, 1ठ, N.

* Phytomyptera cingulata (Robineau-Desvoidy, 1830). Godeliai, 14.VII.-25.VIII.2008, 4우우, M; Karkazai, 28.VII.1999, 1ठ, H; Pamedinčiai, 07.-14.VII.2008, 1ㅇ, M.

*Phytomyptera nigrina (Meigen, 1924). Godeliai, 14.-21.VII.2008, 1q, M; Subartonys, 04.11.VIII.2008, 1ð, M.

**Siphona grandistylum Pandellé, 1894. Eičiai, 26.V.-02.VI.2008, 1ठ, M; Godeliai, 19.V.-

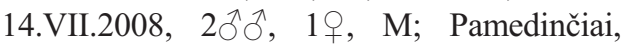
12.V.-09.VI.2008, 5ภㅊ, 4우, M.

*Siphona hokkaidensis Mesnil, 1957. Leipgiriai, 17.VI.-19.VIII.2008, 6 ふे, 19, M.

**Siphona ingerae Andersen, 1982. Eičiai, 14.IV.12.V.2008, 3ठิð, 19, M; Godeliai, 29.III.07.IV.2008, 2ิ̂े, M; Leipgiriai, 21.30.IV.2008, 1ð̄, M; Kleboniškis, 23.V.2006, 1今, N; Lekèčiai, 12.-18.IV.1998, 19, N; Meteliai, 02.-09.IV.2008, 1§, M; Padumblè, 31.III.-14.IV.2008，6今ึ，M; Pamedinčiai, 28.III.-05.V.2008, 4§ึ ô, 4우, M; Subartonys,

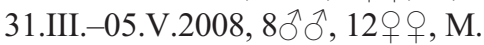

*Siphona paludosa Mesnil, 1960. 2008, 2우, M (data lost).

Solieria inanis (Fallén, 1810). Dūkštos, 29.VII.2006, 1ㅇ, N; Godeliai, 21.VII.-04.VIII.2008, 1ㅇ, M; Leipgiriai, 15.22.VII.2008, 1 , , M.

\subsubsection{Subfamily Exoristinae}

*Admontia grandicornis (Zetterstedt, 1849). Meteliai, 18.-25.VI.2008, 19, M; Pamedinčiai, 30.VI.-08.VII.2008, 1 , , M.

*Atylomya loewi Brauer, 1898. Gruodžiai, 09.VII.2002, 1ठิ, N; 14.VII.2009, 1ठิ, N; Padumblè, 02.-09.VI.2008, 1ð, M.

*Blepharipa schineri (Mesnil, 1939). Markučiai, 27.V.2005, 1ㅇ, N; 10.V.2006, $2 \widehat{\jmath}$, N.

Blondelia inclusa (Hartig, 1838). Eičiai, 05.VIII.08.IX.2008, $2 \hat{\jmath}$ ô, 1 , , M.

* Bothria frontosa (Meigen, 1824). Padumblè, 31.III.-07.IV.2008, $1{ }^{\top}$, M.

*Cadurciella tritaeniata (Rondani, 1859). Leipgiriai, 15.-22.VII.2008, 1ㅇ, M.

**Carcelia puberula Mesnil, 1941. Sakalinè, 
16.V.2000, 1ð̊, N; Želsva, 20.-27.V.2007, 1, M.

**Chetogena tschorsnigi Ziegler, 1999. Vebliai, 15.IV.-13.V.1999, 1ㅇ, P.

* Exorista fasciata (Fallén, 1820). Sakalinè, 10.VI. 1999, $19, \mathrm{~N}$.

* Gastrolepta anthracina Rondani, 1862. Subartonys, 09.-16.VI.2008, 1ㅇ, M.

*Ligeriella aristata (Villeneuve, 1911). Eičiai, 19.-26.VIII.2008, 1ڤ̂, M.

Medina luctuosa (Meigen, 1824). Želsva, 23.VII.2007, 1ㅇ, N.

* Medina melania (Meigen, 1824). Airènai, 24.VI.15.VII.2007, 1今̂, 1ㅇ, M; Čižiūnai, 22.VII. 2007, 1ठ̂, N; Noreikiškès, 28.V.1999, 1ठ, N; Puvočiai, 02.VII.1985, 19, N; Subartonys, 26.V.-28.VII.2008, 1ठิ, 1 , M; Vartai, 08.15.VII.2007, 1, M; Visoriai, 19.VII.2006, $3 \widehat{\partial} \partial^{\lambda}, \mathrm{N}$; Žaizdriai, 14.VII.2007, 19, N.

Meigenia mutabilis (Fallén, 1810). Airenai,

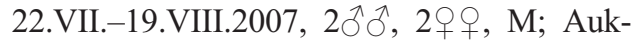
štadvaris, 17.-19.VII.2007, 1ठ, 1, N; Čižiūnai, 22.VII.2007, 1§, N; Dūkštos, 29.VII. 2006, 1ठ, N; Eičiai, 30.VI.-03.IX. 2008, $2 \widehat{\jmath}$, 2오, M; Godeliai, 14.VII.-15.IX.2008, $33 \widehat{\jmath} \widehat{\jmath}, 4$ 웅, M; Gruodžiai, 09.VII.2002, 1ठ, N; 09.VII.2006, 1ð, N; Kleboniškis, 21.VII. 1999, 1ठ, N; Markučiai, 22.VIII.2004, 1ठ, N; Meteliai, 09.-23.VII. 2008, 1ठ઼, M; Padumblè, 04.-10.VIII.2008, 19, M; Pamedinčiai, 23.VI.-18.VIII.2008, 1ðิ, 7우, M; Šeškinè, 11.VIII.2004, 19, N; Vartai, 05.-19.VIII.2007,

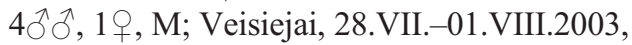
1ठิ, N; Visoriai, 17.VII.-09.VIII.2006, 6§ึ, N; Vyšniūnai, 19.VII.2007, 1ठ̂, N.

* Oswaldia eggeri (Brauer \& Bergenstamm, 1889).

Subartonys, 22.-29.VII.2008, 2우, M.

**Oswaldia reducta (Villeneuve, 1930).

Subartonys, 14.VII.-11.VIII.2008, 2§̂े, M.

Paratryphera bisetosa (Brauer \& Bergenstamm, 1891). Eičiai, 14.VI.2007, 19, N.

**Phebellia pauciseta (Villeneuve, 1908). Markučiai, 17.VI.2006, 1ठ, N.

*Phebellia villica (Zetterstedt, 1838). Airènai, 01.08.VII.2007, 1 , , M.

**Phryxe erythrostoma (Hartig, 1838). Padumblè, 23.-30.VI.2008, 1 , , M.

*Phryxe magnicornis (Zetterstedt, 1838). Eičiai, 15.-29.VII.2008, 1今, 1 우, M.
*Ptesiomyia alacris (Meigen, 1824). Padumblè, 09.-16.VI.2008, 1ð, 1ㅇ, M.

**Senometopia intermedia (Herting, 1960). Želsva, 08.-15.VII.2007, 1 , M.

**Senometopia lena (Richter, 1980). Subartonys, 23.-30.VI.2008, 10, $\mathrm{M}$.

Winthemia erythrura (Meigen, 1838). Visoriai,

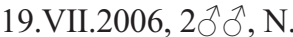

Winthemia venusta (Meigen, 1924). Žaizdriai, 17.VII.2007, 1ठ, N.

\subsection{Host records}

\subsubsection{Subfamily Dexiinae}

Blepharomyia angustifrons Herting, 1971.

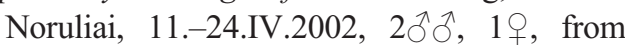
Panolis flammea (Denis \& Schiffermüller) (Lep., Noctuidae).

Thelaira solivaga (Harris, 1780). Žaliakalnis, 22.III.2006, 1今, 1ㅇ, from Phragmatobia fuliginosa (Linnaeus) (Lep., Arctiidae).

\subsubsection{Subfamily Tachininae}

Leskia aurea (Fallén, 1820). Griškabūdis, 29.V.10.VI.1988, 1ठ, from Synanthedon scoliaeformis (Borkhausen) (Lep., Sesiidae).

* Phytomyptera cingulata (Robineau-Desvoidy, 1830). Karkazai, 28.VII.1999, 10, from Nemopogon granella (Linnaeus) (Lep., Tineidae).

\subsubsection{Subfamily Exoristinae}

Aplomya confinis (Fallén, 1820). Marimantas, 02.22.VI.2004, 1ठ, from Satyrium w-album (Knoch) (Lep., Lycaenidae).

Compsilura concinnata (Meigen, 1824). Nida, 23.VII.1995, 1今̄, 1우 from Lymantria dispar (Linnaeus) (Lep., Lymantriidae); Purviniškiai, 27.IX.2007, 1ð, 19, from unidentified Noctuidae (Lep.); Visoriai, 13.VII.2006, 1옹 from Polygonia c-album (Linnaeus) (Lep., Nymphalidae).

Drino inconspicua (Meigen, 1830). Lavysas, 15.19.IV.2010, 3§ิð, 3우, from Dendrolimus pini (Linnaeus) (Lep., Lasiocampidae); Nida, 23.VII.1995, 1§ิ, 2오, from Dendrolimus pini 
(Linnaeus) (ditto); Liškiava, 08.-12.IX.2009, $2 \hat{\jmath}, 3+9$, from Diprion pini (Linnaeus) and D. similis (Hartig) (Hym., Diprionidae).

Huebneria affinis (Fallén, 1810). Eitminiškès, 05.16.XI.2007, 3우, from Phragmatobia fuliginosa (Linnaeus) (Lep., Arctiidae).

Meigenia dorsalis (Meigen, 1824). Želtiškiai, 01.12.VI.2002, 1ㅇ, from Plagiosterna aenea (Linnaeus) (Col., Chrysomelidae).

Meigenia uncinata Mesnil, 1967. Bilšiai, 14.19.VI.2003, 1ð, 2우, from Gonioctena quinquepunctata (Fabricius) (Col., Chrysomelidae).

Nilea rufiscutellaris (Zetterstedt, 1859). Mištautai, 20.IX.-01.X.2006, 1ㅅ, 4우, from Acronicta rumicis (Linnaeus) (Lep., Noctuidae).

Pales pavida (Meigen, 1824). Druskininkai, 24.VI.1996, 10̄, from Panolis flammea (Denis $\&$ Schiffermüller) (Lep., Noctuidae).

Phryxe nemea (Meigen, 1824). Markučiai, 17.27.VI.2006, 1ठ, from Erannis defoliaria (Clerck) (Lep., Geometridae).

\subsection{Deleted records}

\subsubsection{Subfamily Phasiinae}

Gymnosoma costatum: Dumčius \& Pakalniškis (2006). Turned out to be Gymnosoma nitens Meigen, already recorded before.

\subsubsection{Subfamily Tachininae}

Germaria angustata: Pakalniškis \& Podènas (1992). Turned out to be Triarthria setipennis (Fallén), already recorded before.

Loewia phaeoptera: Lutovinovas (2009). Turned out to be the newly described Loewia erecta Bergström (see above).

Phytomyptera bohemica: Rimšaite \& Pakalniškis (2001). Turned out to be Phytomyptera cingulate (Robineau-Desvoidy) (see above).

Siphona rossica: Pakalniškis \& Podėnas (1992). Turned out to be Siphona setosa Mesnil, already recorded before.

Siphona variata: Lutovinovas (2009). Turned out to be Siphona confusa Mesnil, already recorded before.

\subsubsection{Subfamily Exoristinae}

Chetogena fasciata: Lutovinovas (2007). Turned out to be the newly described Chetogena tschorsnigi Ziegler (see above).

Meigenia mutabilis: Lutovinovas et al. (2003). Turned out to be Meigenia uncinata Mesnil (see above).

Tlephusa cincinna: Lutovinovas (2006). Turned out to be Phryxe vulgaris (Fallén), already recorded before.

\section{Discussion}

According to the present revision of the old records, eight species were excluded from the previous species list (Pakalniškis et al. 2006). The true identity of some of them (Chetogena fasciata, Loewia phaeoptera) was clarified after recent discoveries of new species by Ziegler (1999) and Bergström (2007). Some other changes are due to wrong identifications of insects that were corrected after revision of the material.

Out of the species confirmed to occur in Lithuania, 68 species are reported for the first time, which increases the number of species recorded from the area to 276 (Lutovinovas et al. 2003, Lutovinovas 2004, 2006, 2007, 2009, 2010). The primary reason for the high number of new faunistic records from the country during the few recent years is the relatively low intensity of research in the past and the intensive exploration of the regional fauna at present, in particular by means of Malaise traps that are known as the most productive method for collecting the majority of these species (Tschorsnig et al. 2005).

Recent updates to the records of various European countries indicate that many species are expanding their distributional ranges because of the climate change (Bygebjerg 2004, Pohjoismäki 2007, Zeegers 2010). This phenomenon cannot be easily discussed in the East, because the fauna of the Baltic States is insufficiently studied (Tschorsnig et al. 2011). For comparison, the known distribution of several species listed in this paper reaches the Netherlands and Germany in the North, without records in Eastern Europe (Peribaea longirostris, Senometopia lena, etc.), so neither their occurrence in Lithuania was 
straightforwardly expexted nor it claims that these species are expanding their distributional ranges. Some other species are reported exclusively from Central Europe and Finland (Campylocheta mariae, Oswaldia reducta, etc.). In these cases, it was more plausible to expect that these species could occur also in Lithuania. For better understanding of the novelty of my records, it should be noted that 54 species represent first records for the Baltic States as well, whereas 16 are new for Eastern Europe as a whole (former USSR).

Parasitoid-host associations of 13 Tachinidae species with 15 host taxa that comprise 17 couples are recorded for Lithuania for the first time. Among these, Meigenia uncinatata is a new parasitoid of the leaf beetle Gonioctena quinquepunctata (Chrysomelidae). Up to now, M. uncinatata had been considered a specialized parasitoid of Agelastica alni, with its biology described by Mellini (1954).

Acknowledgements. I thank the collectors of the material, and C. Bergström (Uppsala), H.-P. Tschorsnig (Stuttgart) and T. Zeegers (Soest) for checking some specimens. My study was supported by a Research Fellowship of the Czech Science Foundation (GACR 526/09/H025), and also by a grant of the Ministry of Education of the Czech Republic (MSM 0021622416).

\section{References}

Andersen, S. 1996: The Siphonini (Diptera: Tachinidae) of Europe. - Fauna Entomologica Scandinavica 33: 1148.

Bergström, C. 2007: Loewia erecta sp.n. (Diptera: Tachinidae) - a new parasitic fly from Fennoscandia and Poland. - Stuttgarter Beiträge zur Naturkunde Serie A (Biologie) 708: 1-16.

Budrienè, A. 2003: Prey of Symmorphus wasps (Hymenoptera: Eumeninae) in Lithuania. - Acta Zoologica Lituanica 13(3): 306-310.

Bygebjerg, R. 2004: Snyltefluen Phasia hemiptera (Diptera, Tachinidae) genfundet i Danmark. — Entomologiske Meddelelser 72: 75-77.

Bystrowski, C. 2001: A new species of the genus Campylocheta Rondani, 1859 (Diptera: Tachinidae) from Poland. - Annales Zoologici (Warszawa) 51 (3): 279281.

Dumčius, O. \& Pakalniškis, S. 2006: A contribution to the list of Lithuanian Dipteran fauna. - New and Rare for Lithuania Insect Species 17: 50-58.

Herting, B. 1977: Beiträge zur Kenntnis der europäischen Raupenfliegen (Dipt., Tachinidae). - Stuttgarter Beiträge zur Naturkunde Serie A (Biologie) 295: 1-16.
Lutovinovas, E. 2004: Fly (Diptera: Brachycera) species recorded in Lithuania for the first time. - New and Rare for Lithuania Insect Species 16: 62-73.

Lutovinovas, E. 2006: New for Lithuania Calyptratae (Diptera: Muscomorpha) species, recorded in 19261927 and 2002-2006. — New and Rare for Lithuania Insect Species 17: 61-65.

Lutovinovas, E. 2007: Brachycerous fly (Diptera) species new for the Lithuanian fauna. - New and Rare for Lithuania Insect Species 19: 56-62.

Lutovinovas, E. 2009: Tachinidae (Diptera) from the Dūkštų Azžuolynas forest (Neris Regional Park). Dipteron (Wrocław) 25: 38-45.

Lutovinovas, E. 2010: Historical records of Tachinidae (Diptera) from Lithuania. — New and Rare for Lithuania Insect Species 22: 133-136.

Lutovinovas, E., Pakalniškis, S., Petrašiūnas, A. \& Rimšaite, J. 2003: A supplement to the Diptera fauna of Lithuania. -Acta Zoologica Lituanica 13(4): 403 410.

Mellini, E. 1954: Studi sui Ditteri Larvevoridi. II. Meigenia mutabilis Fall. su Agelastica alni L. (Coleoptera, Chrysomelidae). - Rivista di Parassitologia 15: 489512. [In Italian]

Pakalniškis, S. \& Podènas, S. 1992: 258 new to Lithuania Diptera species found in 1964-1992. - New and Rare for Lithuania Insect Species. Records and Descriptions of 1992. Vilnius, 56-82.

Pakalniškis, S., Bernotienė, R., Lutovinovas, E., Petrašiūnas, A., Podėnas, S., Rimšaitè, J., Sæther, O. A. \& Spungis, V. 2006: Checklist of Lithuanian Diptera. New and Rare for Lithuania Insect Species 18: 16150 .

Pohjoismäki, J. 2007: Early season parasitic flies (Diptera: Tachinidae) visiting sap runs on birch trees in Eastern Finland. - The Tachinid Times 20: 1-4.

Rimšaitė, J. \& Pakalniškis, S. 2001: Nauji duomenys apie grybų dvisparnius (Insecta: Diptera) Lietuvoje. - Lietuvos biologinè įvairovẻ (būklè, struktūra, apsauga). Respublikinès konferencijos dalyvių pranešimų santraukos. Vilnius, 75.[In Lithuanian]

Stanionyte, A., Ivinskis, P. \& Pakalniškis, S. 1984: Parasitation of Diptera on Lepidoptera registered in the Lithuanian SSR for the first time. - Acta entomologica Lituanica 7: 118-121. [In Russian.]

Tooker, J. F., Hauser, M. \& Hanks, L. M. 2006: Floral Host Plants of Syrphidae and Tachinidae (Diptera) of Central Illinois. - Annals of the Entomological Society of America 99 (1): 96-112.

Tschorsnig, H.-P. \& Herting, B. 1994: Die Raupenfliegen (Diptera: Tachinidae) Mitteleuropas: Bestimmungstabellen und Angaben zur Verbreitung und Ökologie der einzelenen Arten. - Stuttgarter Beiträge zur Naturkunde, Serie A (Biologie) 506: 1-170.

Tschorsnig, H.-P., Vaňhara, J., Barták, M. \& Kubík, Š. 2005: Tachinidae. - In: Barták, M. \& Kubík, Š. (eds.), Diptera of Podyjí National Park and its Environs: 398-414. Česká zemědělská univerzita, Prague. $432 \mathrm{pp}$.

Tschorsnig, H.-P., Bergström, C., Bystrowski, C., Cerretti, 
P., Hubenov, Z., Raper, C., Van de Weyer, G., Vaňhara, J., Zeegers, T. \& Ziegler, J. 2011: Fauna Europaea: Tachinidae. - In: Pape, T. \& Beuk, P. (eds.), Fauna Europaea: Diptera Brachycera. Fauna Europaea version 2.4. <http://www.faunaeur.org>

Zeegers, T. 2010: Tweede aanvulling op de naamlijst van Nederlandse sluipvliegen (Diptera: Tachinidae). Nederlandse faunistische mededelingen 34: 55-66. [In Dutch]
Ziegler, J. 1999: Eine neue paläarktische Art aus der Raupenfliegengattung Chetogena (Diptera: Tachinidae). - Studia dipterologica 6(2): 437-444.

Ziegler, J. \& Shima, H. 1996: Tachinid flies of the Ussuri area (Diptera: Tachinidae). — Beiträge zur Entomologie 46: 379-478. 